\begin{tabular}{l|l|l|l|l|}
$\begin{array}{l}\text { Journal of } \\
\text { Endocrinology }\end{array}$ & L Fu, Y Qiu et al. & $\begin{array}{l}\text { Postpone impacts of antibiotics } \\
\text { on db/db mice }\end{array}$ & $\mathbf{2 3 8 : 2}$ & $\mathbf{1 3 7 - 1 4 9}$ \\
\hline
\end{tabular}

RESEARCH

\title{
The delayed effects of antibiotics in type 2 diabetes, friend or foe?
}

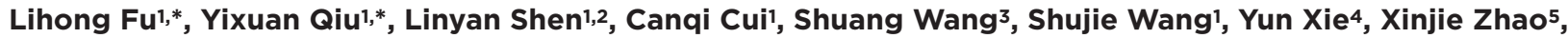 \\ Xianfu Gao6, Guang Ning', Aifang Nie ${ }^{1}$ and Yanyun Gu'
}

\begin{abstract}
1Shanghai National Research Center for Endocrine and Metabolic Diseases, Shanghai Institute for Endocrine and Metabolic Diseases, Ruijin Hospital, Shanghai Jiao Tong University School of Medicine, Shanghai, China

2Department of Endocrinology, East Hospital, Tongji University School of Medicine, Shanghai, China

3BGI Genomics, BGI-Shenzhen, Shenzhen, China

${ }^{4}$ Department of Cardiology, Ruijin Hospital, Shanghai Jiao Tong University School of Medicine, Shanghai, China

${ }^{5}$ Key Laboratory of Separation Science for Analytical Chemistry, Dalian Institute of Chemical Physics, Chinese Academy of Sciences, Dalian, China

${ }^{6} \mathrm{Key}$ Laboratory of Systems Biology, Institute of Biochemistry and Cell Biology, Shanghai Institutes for Biological Sciences, Chinese Academy of Sciences,

Shanghai, China
\end{abstract}

Correspondence should be addressed to A Nie or Y Gu: afnie@126.com or guyanyun@hotmail.com

*(L Fu and Y Qiu contributed equally to this work)

\section{Abstract}

An increasing amount of evidence suggests that the delayed effect of antibiotics (abx) on gut microbiota after its cessation is not as favorable as its immediate effect on host metabolism. However, it is not known how the diverse abx-dependent metabolic effects influence diabetic subjects and how gut microbiota is involved. Here, we treated $\mathrm{db} / \mathrm{db}$ mice with abx cocktail for 12 days and discontinued for 24 days. We found that $\mathrm{db} / \mathrm{db}$ mice showed decreased body weight and blood glucose after abx treatment, which rapidly caught up after abx cessation. Twenty-four days after abx withdrawal, $\mathrm{db} / \mathrm{db}$ mice exhibit increased plasma, hepatic total cholesterol (TC) levels and liver weight. The gut microbiota composition at that time showed decreased relative abundances (RAs) of Desulfovibrionaceae and Rikenellaceae, increased RA of Erysipelotrichaceae and Mogibacteriaceae, which were correlating with the reduced short-chain fatty acids (SCFAs) in gut content, such as propionic acid and valeric acid and with the elevated fecal taurine-conjugated bile acids (BAs) levels. The molecular biology studies showed inhibited hepatic BA synthesis from cholesterol, impeded intracellular transportation and biliary excretion of cholesterol that all conferred to liver TC accumulation. The associations among alterations of gut microbiota composition, microbial metabolite profiles and host phenotypes suggested the existence of gut microbiota-linked mechanisms that mediate the unfavorable delayed effects of abx on $\mathrm{db} / \mathrm{db}$ mice cholesterol metabolism. Thus, we call upon the caution of applying abx in diabetic animal models for studying microbiota-host interaction and in type 2 diabetes subjects for preventing chronic cardiovascular consequences.
Key Words
- gut microbiota
- bile acids
- antibiotics
- type 2 diabetes
- cholesterol 


\section{Introduction}

It has been well established that the gut microbiome affects host metabolic homeostasis, and gut dysbiosis promotes the development of multiple human metabolic disorders, such as obesity, non-alcoholic fatty liver disease and type 2 diabetes mellitus (T2D) (Turnbaugh et al. 2006, Qin et al. 2012, Ridaura et al. 2013, Lynch \& Pedersen 2016). Ablating gut microbiota, as in germ free (GF) mice, improves glucose tolerance and renders mice resistant to diet-induced obesity (DIO) (Rabot et al. 2010). On the other hand, treatment with single or cocktail of antibiotics (abx), wildly applied to achieve the status mimic 'germ free' in conventionally raised animals, has been proved to improve the host metabolism, regardless of different mouse strain backgrounds, types and lengths of abx interventions (Carvalho et al. 2012, Hwang et al. 2015, Fujisaka et al. 2016).

Interestingly, evidence is accumulating to suggest that some effects of transient abx exposures are long lasting, particularly in early life, which can alter the gut microbiome structure and hence compromise the host metabolism and immunity development in later life, associating with the increased risks of metabolic disorders and autoimmune diseases (Cho et al. 2012, Cox et al. 2014, Livanos et al. 2016). A retrospective study on a large cohort in Denmark showed that the exposure of antibiotics is positively associated with the risk of T2D (Mikkelsen $e t$ al. 2015b). Recent longitudinal studies observing long-lasting effects after 7-day single abx treatment in healthy adults (Mikkelsen et al. 2015a, Reijnders et al. 2016), have found no metabolic improvements or even a few of metabolic disturbances. Thus, it will be of interest to study how the dual effects of antibiotics treatment, in terms of the transient (immediate) or long-lasting influences, are exerted on the gut commensal microbiota and the host metabolic consequences in T2D.

Instead of depleting the whole gut microbiota, abx treatment actually reshapes the whole intestinal ecology by suppressing common commensal obligate anaerobes such as phylum Bacteroides, and expanding facultative anaerobic species that rarely occur in the normal condition, such as phylum Proteobacteria (Fujisaka et al. 2016, Ianiro et al. 2016, Byndloss et al. 2017). These changes in gut microbiome, however, rather than recovering gut microbiota symbiosis after abx cessation, often lead to a different status of microbiota dysbiosis. The taxa changed after transient abx treatment include those short-chain fatty acids (SCFAs) producers and/or bile acids (BAs) convertors, hereafter, the transient abx treatment also disturbs the production of these main bacteria metabolites (Jiang et al. 2015a, Livanos et al. 2016). However, it is less clear if SCFAs or BAs also exhibit the alterations that are different from the assumed 'recovery' pattern after abx cessation.

It has been proved the effects that gut microbiome exerts on host metabolism require BA signaling (Parseus et al. 2017) or SCFAs (Cho et al. 2012, Reijnders et al. 2016) as well. And abx-treated mice show similar BA profiles as those of GF mice, with increased taurine-conjugated and primary BAs, taurine-conjugated beta murine cholic acid (TBMCA) in particular (Sayin et al. 2013, Jiang et al. 2015a). These changes of BAs are driven by the disputed bacterial BA transformation pathway, which is manifested by decreased abundancies of bacterial bile salt hydrolase (Bsh) and bile acid-inducible (Bai) genes that are contained mainly by the taxa of Bacteroidia and Clostridia. By sensing the BA alterations, the nucleus BA receptor, Fxr (nuclear receptor subfamily 1, group $\mathrm{H}$, member 4, Nr1h4) governs the BA pool size, composition and regulates metabolic homeostasis (Wahlstrom et al. 2016) in a compartmentation-dependent manner. Following with the 'SCFAs producers' in the microbiome upon abx treatment (Cho et al. 2012, Reijnders et al. 2016), the intraluminal SCFAs levels change to promote host metabolic health, though the roles of SCFAs in regulating host metabolism in other conditions have controversial conclusions (De Vadder et al. 2014, Rios-Covian et al. 2016, Murugesan et al. 2018). Thus, it will be of value to investigate how BA signaling or SCFAs contribute to the long-lasting abx effects on metabolic regulation in diabetic conditions.

In this study, to address the delayed effects of abx exposure on metabolic and gut microbiota in T2D, we treated $\mathrm{db} / \mathrm{db}$ mice with a cocktail mix of four kinds of abx for 12 days and withdraw for another 24 days. In contrast to the improved metabolic status in $\mathrm{db} / \mathrm{db}$ mice after transient abx treatment, the mice increased BW gain and cholesterol levels after abx withdrawing. The decreased Bacteroides and increased Firmicutes were manifested as the gut microbiota dysbiosis after abx cessation and correlated with increased fecal taurine BAs and decreased odd carbon SCFAs. Together, the altered taxonomy and metabolites correlate closely with the host unfavorable phenotypes. Finally, with the altered expressions of key genes regulating BA synthesis/excretion and cholesterol transportation after abx cessation, we identified potential microbiota-host interaction pathways that could improve the metabolic status in $\mathrm{db} / \mathrm{db}$ mice by promoting liver cholesterol metabolism. 


\section{Materials and methods}

\section{General animal housing and maintenance}

Four- to six-week-old male C57BKS-Lepr-1- (db/db) mice were purchased from the Model Animal Research Center of Nanjing University. Mice were fed ad libitum and housed at $23 \pm 1^{\circ} \mathrm{C}$ with a relative humidity of $55 \pm 5 \%$ for 12-h light/darkness switch. All the experimental protocols were approved by the Animal Use and Care Committee of Shanghai Jiao Tong University.

\section{Antibiotic treatment}

Mice were randomly divided into control (Veh) and antibiotics (Abx) group upon purchase. The Veh group mice were subjected to drinking water only throughout the experiment, and the Abx group treated with antibiotic cocktail containing ampicillin $(1 \mathrm{~g} / \mathrm{L})$, neomycin $(1 \mathrm{~g} / \mathrm{L})$, streptomycin $(1 \mathrm{~g} / \mathrm{L})$ and doxycycline $(1 \mathrm{~g} / \mathrm{L})$ in the drinking water for 12 days and then discontinued for another 24 days before euthanasia.

\section{Glucose and insulin tolerance tests}

Mice were subjected to an oral glucose tolerance test (OGTT) after an overnight fasting. An oral gavage of glucose solution at the dose of $2 \mathrm{mg} / \mathrm{g}$ (weight/body weight) was given. Blood was collected from orbital venous plexus 0,15 and $30 \mathrm{~min}$ after glucose gavage in the tubes pretreated with EDTA-Na ${ }^{+}$and pre-added DPPIV inhibitor (Merck Millipore). Plasma was immediately separated at $4^{\circ} \mathrm{C}$ by centrifuging and stored at $-80^{\circ} \mathrm{C}$ until further analysis.

For insulin tolerance test (ITT), mice were injected intraperitoneally with human insulin $2 \mathrm{IU} / \mathrm{kg}$ body weight (Novo Nordisk) after 6-h fasting. Blood glucose was measured by tail bleeding at $0,15,30$ and $60 \mathrm{~min}$ after the injection.

\section{Body composition}

Body composition (fat mass and lean mass) was assessed by an animal whole body composition analyzer (EchoMRI $100 \mathrm{H}$, Houston, TX, USA) according to the manufacturer's instructions. Before euthanasia, all the mice were fasted overnight and anesthetized with 10\% chloral hydrate. Blood was collected in tubes containing EDTA-Na+ and DPPIV inhibitor. Liver, intestine and epidydimal white adipose tissue were dissected, weighed and then snap frozen at liquid nitrogen and stored at $-80^{\circ} \mathrm{C}$ for further analysis.

\section{Quantitative real-time PCR}

Total tissue RNA was extracted by TRIzol reagent (Invitrogen) in accordance with the manufacturer's protocols and quantified by NanoDrop 2000 spectrophotometer, $1 \mu \mathrm{g}$ RNA was used for reverse transcription with the Reverse Transcription System Kit (Promega) to cDNA. Real-time PCR amplification and detection was performed using the SYBR Green II Master (Takara) on LightCycler 480 (Roche Applied Science). The expression levels were normalized to housekeeping 36b4 gene. The primers used in this study are listed in (Supplementary Table 1, see section on supplementary data given at the end of this article).

\section{Western blotting}

Proteins were extracted from tissues with RIPA buffer (Cell Signaling Technology) in tissue processor and quantified using assay Pierce BCA Protein Assay Kit (Thermo Scientific) according to the manufacturer's guide. After protein was separated by SDS-PAGE and subjected to electrophoretic transfer on nitrocellulose membranes that were blotted with primary antibodies: NPC1 (1:1000) from Proteintech (Chicago, IL, USA), FXR (1:1000), small heterodimer partner (SHP) (1:1000) from Santa Cruz and $\alpha$-tubulin (1:1000) from Cell Signaling Technology. The blotting bands were illuminated by Immobilon Western HRP (Merck Millipore) and visualized with Image Quant LAS 4000 (GE Healthcare Life Sciences).

\section{Cholesterol and triglycerides measurement}

Plasma triglyceride (TG) and total cholesterol (TC) were determined by colorimetric assay kit (Biovision) according to the manufacturer's protocols. For hepatic lipids measurement, liver tissues were homogenized in Folch solution (chloroform/methanol, 2:1, v/v) and the tissue lysates and plasma samples were subjected to colorimetric enzymatic assay using TC or TGs colorimetric assay kit (Biovision) according to the manufacturer's instructions.

\section{Laboratory assays for incretins and bacterial metabolites}

Metabolic hormone levels in plasma were assayed by a Luminex FlexM3D system with xMAP Luminex technology (Merck Millipore). BAs were extracted from plasma, feces and liver using methanol-containing internal standards and analyzed using ultra-performance 
liquid chromatography-tandem mass spectrometry (UPLC-MS/MS) as described previously (Gu et al. 2017). SCFAs were extracted from cecum samples and analyzed by GC-MS metabolomics using an Agilent 7890A gas chromatography system coupled to an Agilent 5975C inert MSD system (Agilent Technologies Inc.). Data were analyzed with MSD ChemStation software (E.02.02.1431, Agilent Technologies, Inc.). The standards were purchased from Aladdin.

\section{$16 S$ rRNA gene sequencing of the gut microbiota}

DNA was extracted from frozen feces as previously described (Li et al. 2014). The v3-v4 region of the bacteria $16 \mathrm{~S}$ ribosomal RNA gene was amplified by PCR. The melting temperature is $56^{\circ} \mathrm{C}$ and PCR cycle is 30, using primers 341F 5'-barcode-ACTCCTACGGGAGGCAGCAG-3' and 806R 5'-GGACTACHVGGGTWTCTAAT-3', where barcode is an eight-base sequence unique to each sample. The qualified libraries will be sequenced pair end on the MiSeq System (Illumina, Inc.), with the sequencing strategy PE250 (PE251+8+8+251) (MiSeq Reagent Kit). The high-quality paired-end reads were combined to tags when the two paired-end reads overlapped, using FLASH (Fast Length Adjustment of Short reads, v1.2.11) (Magoc \& Salzberg 2011). Taxonomic ranks were assigned to operational unit taxonomy (OTU) representative sequence using Ribosomal Database Project Classifier v.2.2 trained on the Greengenes database, using 0.6 confidence values as cutoff. Unassigned OTUs and OTUs not assigned to the target species were removed. The filtered OTUs were used to downstream processing. At last, the different species screening was analyzed based on OTU and taxonomic ranks.

\section{Statistical analysis}

Statistical calculations were performed with Student's t-test to detect differences between two groups' data of metabolic parameters using SPSS 11.0 software (SPSS Inc.). Kruskal-Wallis test was also used to detect differences in the RAs of gut microbiota, levels of BAs and SCFAs comparison between groups in $\mathrm{R}$ software (3.4.0.). The Spearman's correlation analysis calculated by $\mathrm{R}$ 3.4.0, and the heatmap of clustering of correlation coefficients was calculated by hierarchical Ward and the heatmap was plotted by R. FDR was calculated from the $P$ values adjusted by the Benjamini-Hochberg correction for multiple testing using $\mathrm{R}$ software (v3.4.0). All data was shown as mean \pm s.e.M. and $P$ value $<0.05$ was generally considered to be statistically significant.

\section{Results}

The transient and long-lasting effects of antibiotics on metabolic phenotypes of $\mathrm{dlb} / \mathrm{dlb}$ mice

Six-week-old male $\mathrm{db} / \mathrm{db}$ mice received a cocktail mix of abx for 12 days and withdraw for another 24 days (Fig. 1A). The mice showed a trend of decreased BW and significant reduced random blood glucose (BG) for 12-day abx treatment compared to the mice treated by vehicle (Veh) (Fig. 1B and D; $P<0.05$ ). And the Veh groups showed significantly larger BW gain and BG increments (Fig. 1C and E; $P<0.05)$ than Abx group after 12-day abx treatment. All these differences disappeared 24 days after abx withdrawal (Fig. 1B and D), while the total BW gain after abx withdrawal was significantly higher in the mice pretreated with abx (p-Abx) vs mice consistently treated with vehicle (p-Veh) (Fig. 1C). There was no significant difference in the BG changes, fasting BG, insulin tolerance between p-Abx and p-Veh groups (Fig. 1E, F and G). The levels of plasma insulin and incretins during an OGTT, including GLP-1, GIP and PYY, also showed no differences between the groups (Fig. 1H and Supplementary Fig. 1). The p-Abx mice had higher epidydimal fat mass and liver weight compared to the p-Veh mice with similar body composition (Fig. 1I, J and $\mathrm{K}$ ). The liver histology did not exhibit differences in hepatic lipid droplets between the p-Abx and p-Veh groups (Supplementary Fig. 2), neither did the hepatic TG content, but the levels of TC in liver and plasma were significantly higher in p-Abx mice (Fig. 1L and M). In summary, the beneficial metabolic outcomes during abx treatment in $\mathrm{db} / \mathrm{db}$ mice were mainly found to be reversed after abx withdrawal along with the resilience of suppressed food intake (Supplementary Fig. 3), except for the higher plasma and liver cholesterol levels after abx cessation.

The transient and long-lasting effects of antibiotics on gut microbiota compositions in $\mathrm{db} / \mathrm{dlb}$ mice

We then analyzed the changes of fecal microbiota composition by $16 \mathrm{~S}$ rRNA gene amplicon sequencing. The principal component analysis (PCA) at OTU level clearly separated the mice treated with vehicle, abx treatment and abx withdrawal (Fig. 2A). And histograms of class, order and genus levels indicated that abx treatment dramatically 


\begin{tabular}{l|l|l|c|}
$\begin{array}{l}\text { Journal of } \\
\text { Endocrinology }\end{array}$ & $\begin{array}{l}\text { Postpone impacts of antibiotics } \\
\text { on db/db mice }\end{array}$ & $\mathbf{2 3 8 : 2}$ & $\mathbf{1 4 1}$ \\
\hline
\end{tabular}

A

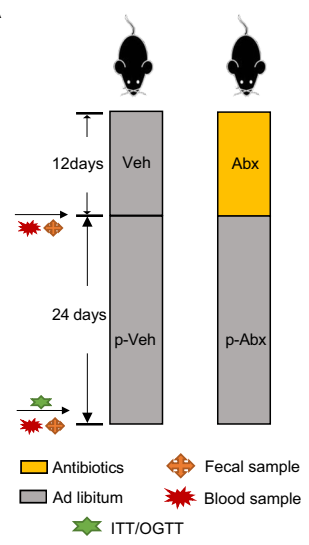

$\mathrm{F}$
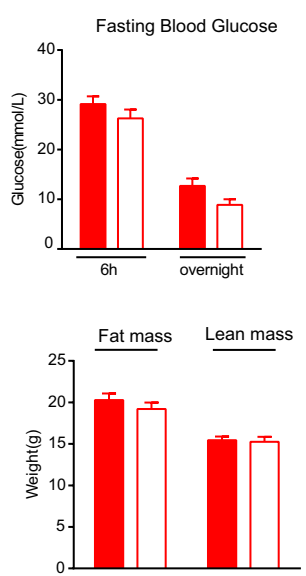

B
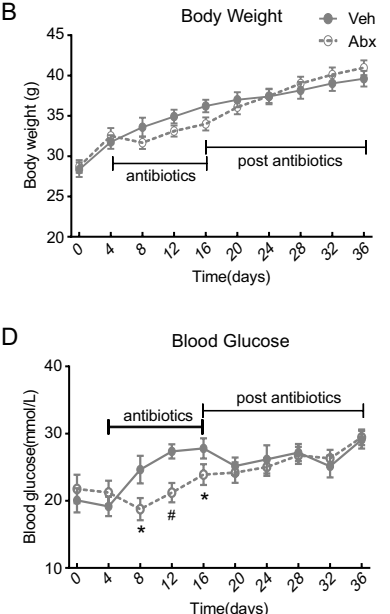

G

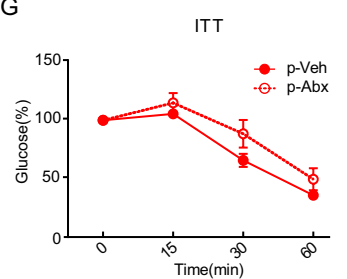

J

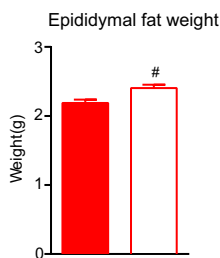

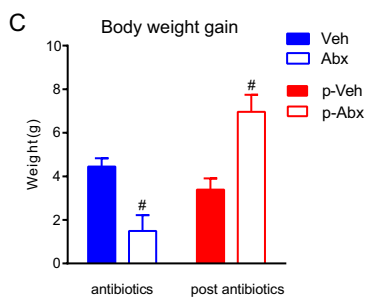

E

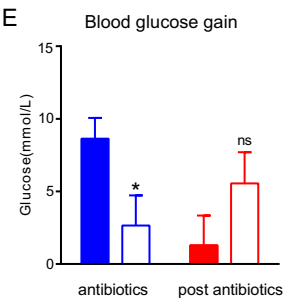

$\mathrm{H}$

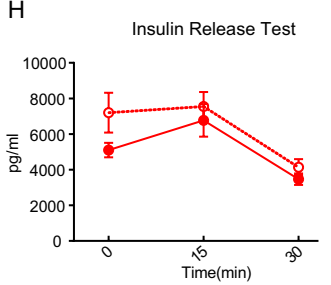

K

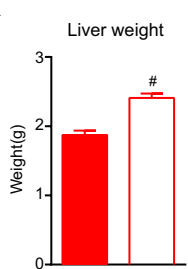

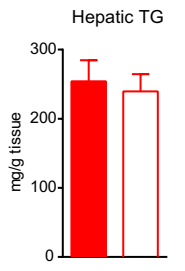

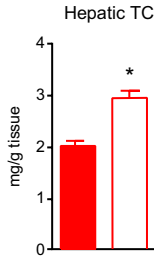

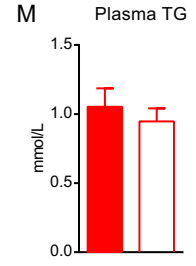

\section{Figure 1}

Changes of obesity and diabetic-related

phenotypes. (A) Scheme of study design. (B) Time curve of body weight changes during antibiotics treatment and after antibiotics withdrawal. (C) The transient and prolonged effects of antibiotics treatment on body weight gain, $n=10-12$. (D) Time curve of random blood glucose alterations during antibiotics treatment and after antibiotics withdrawal. (E) The transient and prolonged effects of antibiotics treatment on random blood glucose increment, $n=10-12$. (F) Fasting blood glucose after antibiotics withdrawal. Six-hour fasting, $n=4-5$ and overnight fasting $n=8$. (G) Insulin tolerance test (ITT) after antibiotics withdrawal. The percentage of the blood glucose change from basal level after insulin intraperitoneal injection. $n=4-5$ for each group. (H) Insulin release test (IRT) after antibiotics withdrawal. The plasma insulin levels before (0 min), $15 \mathrm{~min}$ and $30 \mathrm{~min}$ of OGTT, $n=6-8$ for each group. (I) Body composition, (J) epidydimal fat and $(K)$ liver weight after antibiotics withdrawal, $n=6-8$. (L) Hepatic TG and TC content after antibiotics withdrawal. $n=4-5$ for each group. (M) Plasma TG and TC levels after antibiotics withdrawal. $n=6-8$ for each group. Abx, antibiotics-treated mice; Veh, vehicle-treated mice; $p$-Abx, mice treated with antibiotics for 12 days and withdrawal for 24 days; $p$-Veh, mice treated with vehicle in two stages. TG, triglycerides; TC, total cholesterol. Data are presented as mean \pm S.E.M., ${ }^{*} P<0.05, \# P<0.01, \mathrm{Abx}$ vs Veh or p-Abx vs $p$-Veh, Student's $t$-test. altered the microbiota composition and the immediate response of microbiome to abx can be reversed in some degree after 24-day abx withdrawal but be still different with those of vehicle groups (Supplementary Fig. 4A, $\mathrm{B}$ and $\mathrm{C}$ ). The relative abundances (RAs) of both phyla Firmicutes and Proteobacteria have markedly increased and those of phyla Bacteroidetes and Deferribacteraceae decreased by 12-day abx treatment. After 24-day withdrawal, most above taxonomic changes reversed, but the phyla Bacteroidetes and Firmicutes in p-Abx group could not recover to the control levels $(P<0.05$, Fig. 2B and Supplementary Fig. 4D). Of note, some taxa with low abundancies or rare occurrences in Veh groups were enriched significantly by abx treatment and dropped down after the abx cessation. In the family level (Fig. 2C),
Desulfovibrionaceae (Proteobacteria) and Rikenellaceae (Bacteroidetes) showed even less abundance, and families Erysipelotrichaceae and Mogibacteriaceae under phylum Firmicutes were more abundant after abx withdrawal than those immediately after abx treatment.

Of note, we found that gut microbiota, which reacted similarly to the long-lasting effects of abx, were also clustered together according to their correlations with the main metabolic parameters (Fig. 2D). The hepatic, plasma TC levels and BW gain were all negatively correlated with the RAs of Desulfovibrionaceae and Rikenellaceae. The RA of Mogibacteriaceae was positively correlated with plasma TC and BW gain. The families of Bacteroidaceae, Peptostreptococcaceae and Clostridiaceae correlated positively with BG levels and 


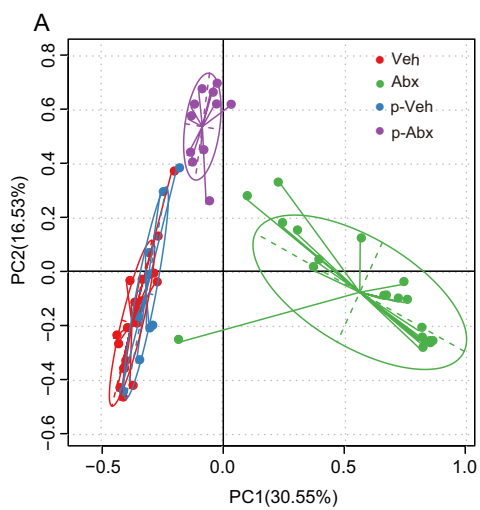

B

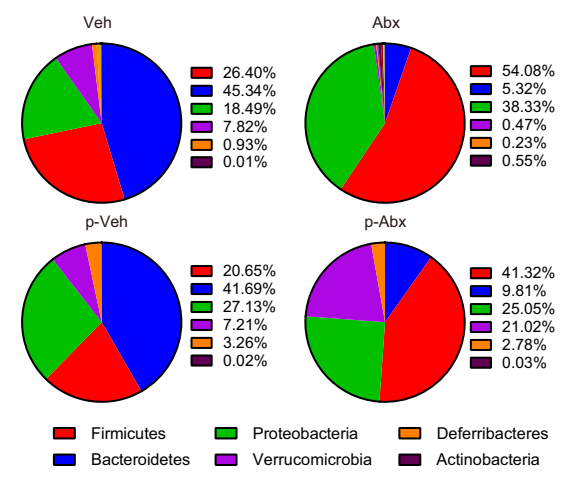

C

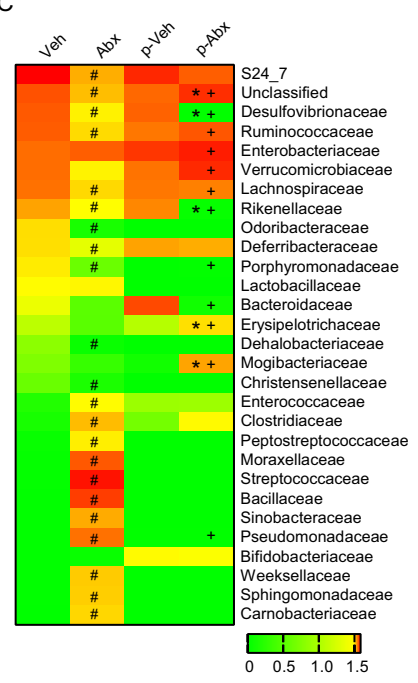

D

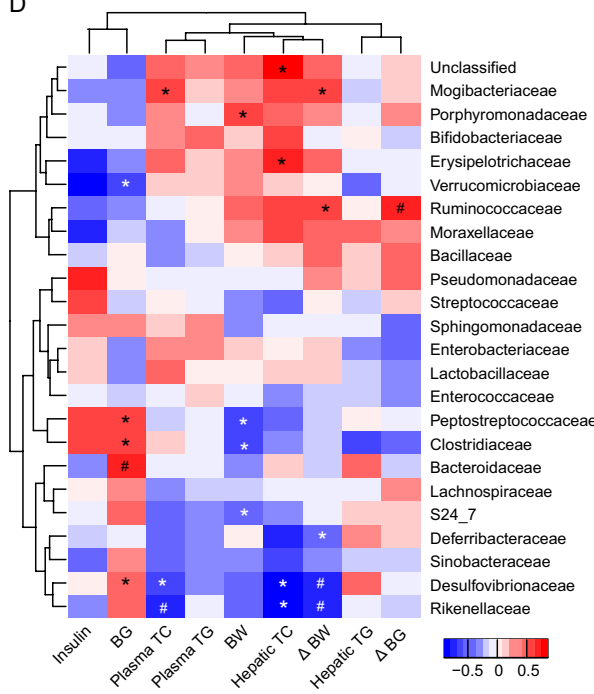

Figure 2

The transient and long-lasting impact of antibiotics treatment on Gut microbiota composition. (A) Principal component analysis (PCA) analysis based on OTUs composition. Each dot represents an individual. The first two components PC1 and PC2 are plotted in $X$ and $Y$ axis, where the percentage of variation explained by each principal component is indicated. (B) Pie plots representing the gut microbiota composition at phylum levels. $n=10-20$ for each group. (C) Heat map of gut microbiota composition at family levels. Log transformed relative abundances that were classified at that rank. $n=10-20$ for each group. *FDR $<0.05$, ${ }^{*} F D R<0.01, A b x$ vs Veh and $p$-Abx vs $p$-Veh, $+F D R<0.05, p-A b x$ vs Abx, Kruskal-Wallis test. (D) The heatmap of Spearman's correlation analysis between relative abundances (RAs) of bacterial families and mice phenotypes. The color present Spearman's correlation co-efficiency: red colors indicate positive relationship, blue colors indicate negative relationship. $n=8-10$ for each group. ${ }^{*} P<0.05,{ }^{P} P<0.01$, Abx vs Veh and $p$-Abx vs $p$-Veh, Spearman's correlation. the latter two negatively associated with the BW. Thus, the long-lasting abx effects on microbiota might be associated with metabolic disorders, including BW gain, TC levels and BG changes.

\section{Effects of transient and long-lasting abx on main bacterial metabolites}

We next analyzed the prolonged impact of abx on microbial metabolites, BAs and SCFAs. We first determined and quantified the BAs in different compartments before and after abx withdrawal. Though the changes of total amount of BAs in both plasma and feces were not significant (Supplementary Fig. 5A and B), the clear separation of BA compositions between vehicle and abx treatment (Fig. 3A) disappeared after abx withdrawal (Fig. 3B). In line with previous studies, the abx reduced the diversity of BAs in both feces and plasma (Fig. 3C and Supplementary Fig. 5C), but elevated the ratio of primary BAs to secondary BAs (PBA/SBA), conjugated to unconjugated BAs
(CBA/UCBA), $12 \alpha$-hydroxylated to non $12 \alpha$-hydroxylated BAs (12 $\alpha \mathrm{BA} /$ non $12 \alpha \mathrm{ba})$ (Fig. 3D), and levels of taurineconjugated BA species (taurine BAs) (Fig. 3E), T $\beta$ MCA in particular (Fig. 3F). Though most BA composition changes were recovered after 24-day withdrawal of abx (Fig. 3G, $\mathrm{H}$ and Supplementary Fig. 5D, E), the taurine BAs\%, T $\beta \mathrm{MCA} \%$, taurine-conjugated deoxycholic acid (TDCA) $\%$ and reduced omega murine cholic acid ( $\omega \mathrm{MCA}) \%$ in fecal BA pool of p-Abx mice were significantly elevated compared to those of the p-Veh group (Fig. 3I, J and Supplementary Fig. 5D), and the plasma T $\beta \mathrm{MCA} \%$ was even increased after abx cessation. We next compared the SCFAs levels in the cecum samples from mice of $\mathrm{p}$-Abx and $\mathrm{p}$-Veh. The SCFAs showed no changes in total amount (Fig. 3K), nor did the levels of acetate and butyrate (Fig. 3L) in $\mathrm{p}$-Abx compared to $\mathrm{p}$-Veh. However, the propionic and valeric acid levels were significantly reduced in the $\mathrm{p}-\mathrm{Abx}$ group (Fig. 3L).

Interestingly, we found levels of fecal primary taurine BA species and taurine conjugated ursodeoxycholic acid 


\begin{tabular}{l|l|l|l|l|}
$\begin{array}{l}\text { Journal of } \\
\text { Endocrinology }\end{array}$ & L Fu, Y Qiu et al. & $\begin{array}{l}\text { Postpone impacts of antibiotics } \\
\text { on db/db mice }\end{array}$ & $\mathbf{2 3 8 : 2}$ & $\mathbf{1 4 3}$ \\
\hline
\end{tabular}
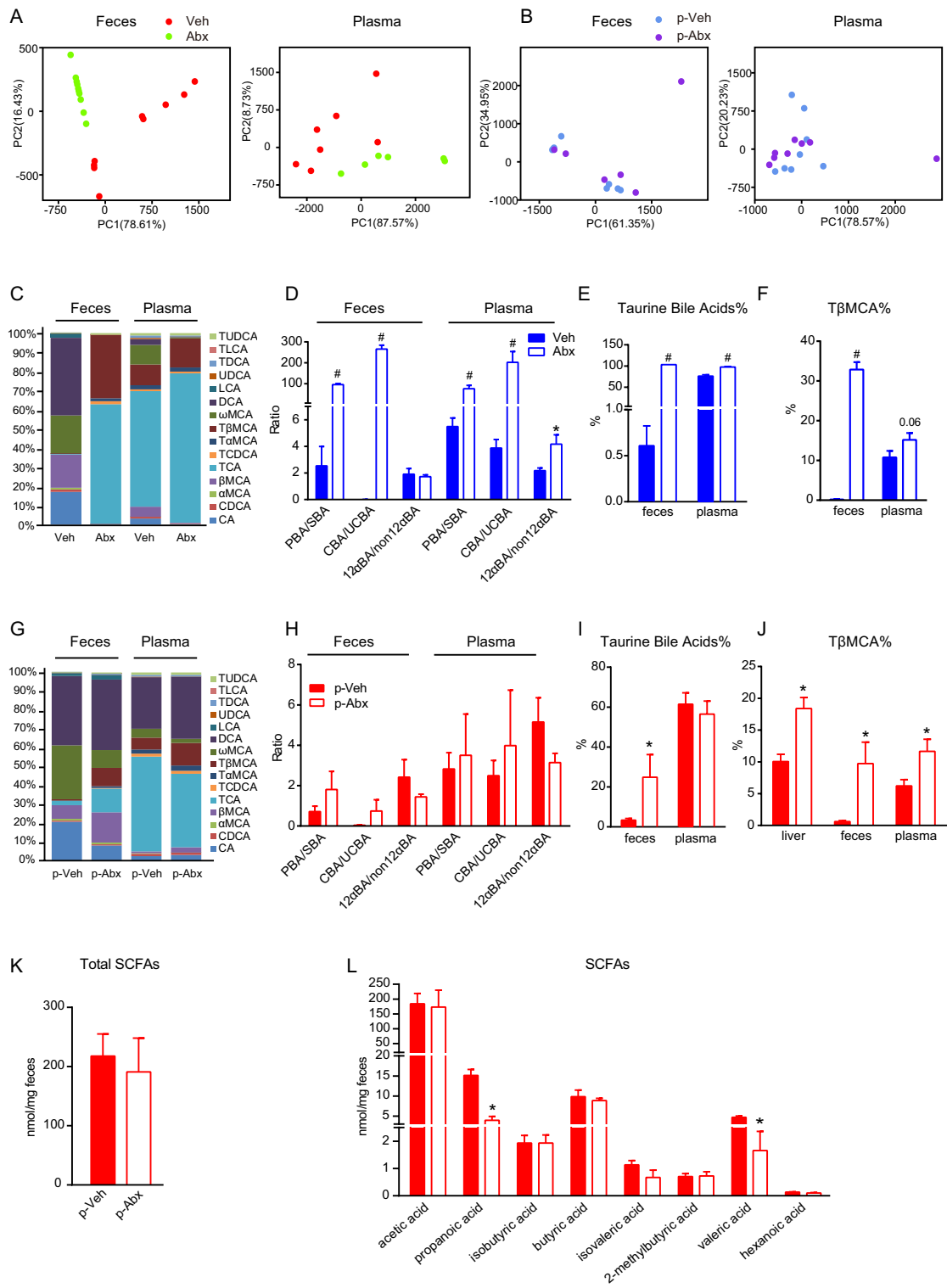

Figure 3

The transient and long-lasting impact of antibiotics treatment on feces and plasma bile acids (BAs) composition. PCA analysis of feces and plasma bile acid (BAs) (A) during abx treatment and (B) after abx withdrawal. (C) The stacking chart of feces and plasma BAs composition during abx treatment. (D) The ratio of different components of feces and plasma BAs during abx treatment. Percentage of (E) Taurine BAs, and (F) T $\beta M C A$ in feces and plasma during abx treatment. (G) The percentage stacking chart of feces and plasma BAs after abx withdrawal. (H) The ratio of different components of feces and plasma BAs after antibiotics withdrawal. Percentage of (I) Taurine BAs, and (J) TBMCA in feces and plasma after abx withdrawal. (K) Total short-chain fatty acid (SCFA) levels in cecum after abx withdrawal. (L) The compositions of total SCFA in cecum after abx withdrawal. $n=6-10$ for each group. $C A$, cholic acid; CDCA, chenodeoxycholic acid; MCA, murine cholic acid; $\alpha M C A$, alpha MCA; $\beta$ MCA, beta MCA; TCA, taurine conjugated cholic acid; TCDCA, taurine conjugated chenodeoxycholic acid; TळMCA, taurine conjugated alpha MCA; T $\beta M C A$, taurine conjugated beta MCA; $\omega M C A$, omega MCA; DCA, deoxycholic acid; LCA, lithocholic acid; UDCA, ursodeoxycholic acid; TDCA, taurine conjugated deoxycholic acid; TLCA, taurine conjugated lithocholic acid; TUDCA, taurine conjugated ursodeoxycholic acid; taurine $B A s$, taurine conjugated BAs; PBA, primary BAs; $S B A$, secondary $B A s ; C B A$, conjugated $B A s ; U C B A$, unconjugated $B A s ; 12 \alpha B A, 12 \alpha$-hydroxylated BAs; non12 $\alpha \mathrm{BA}$, non $12 \alpha$-hydroxylated BAs. Data are showed as mean \pm S.E.M., ${ }^{*} P<0.05,{ }^{\#} P<0.01$, Abx vs Veh and $p$-Abx vs $p$-Veh, Kruskal-Wallis test.
(TUDCA) were clustered to positively correlate with the levels of hepatic, plasma cholesterol and BW gain (Fig. 4A, Spearman correlation, $P<0.05)$. Whereas the cecum propionic and valeric acids were negatively correlated with plasma TC, the propionic acid negatively with BW gain and valeric acids negatively with hepatic TC accumulation, respectively (Fig. 4A, Spearman correlation, $P<0.05$ ). Few of the plasma BAs were significantly associated with changed metabolic parameters and gut microbiota, except for the correlations between plasma taurine BAs with BG and with BG-related taxa (Supplementary Fig. 6A and B, Spearman correlation, $P<0.05)$. Different microbiota data clustered based on correlation with phenotypes also shared similar associations with the bacterial-related metabolites (Fig. 4B, Spearman correlation, $P<0.05)$. For example, the Desulfovibrionaceae and Rikenellaceae both positively correlated with propionic acid and valeric acid levels simultaneously had negative correlation with the fecal taurine BA species (Fig. 4B, Spearman correlation, $P<0.05$ ). Besides, Erysipelotrichaceae and Mogibacteriaceae were both positively correlated with deoxycholic acid (DCA) and lithocholic acid (LCA), the main SBA species in feces.

Of note, though no significant changes in BG levels or changes after abx withdrawal, some of the plasma taurine BAs, cecum acetic and hexanoic acids levels were positively correlated with the BG levels. The BG-related taxa, the families of Bacteroidaceae, Peptostreptococcaceae and Clostridiaceae also share similar positive connections with these above metabolites (Fig. 4B, Spearman correlation, $P<0.05)$. Thus, these results suggested the long-lasting 


\begin{tabular}{l|l|l|r|r|}
$\begin{array}{l}\text { Journal of } \\
\text { Endocrinology }\end{array}$ & L Fu, Y Qiu et al. & $\begin{array}{l}\text { Postpone impacts of antibiotics } \\
\text { on db/db mice }\end{array}$ & $\mathbf{2 3 8 : 2}$ & $\mathbf{1 4 4}$ \\
\hline
\end{tabular}

A

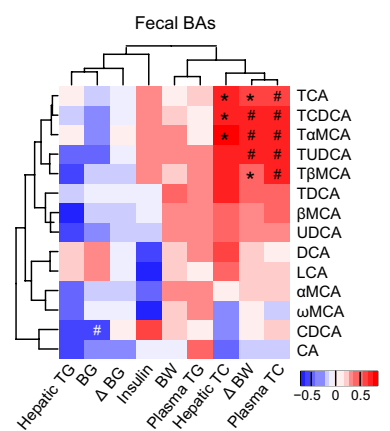

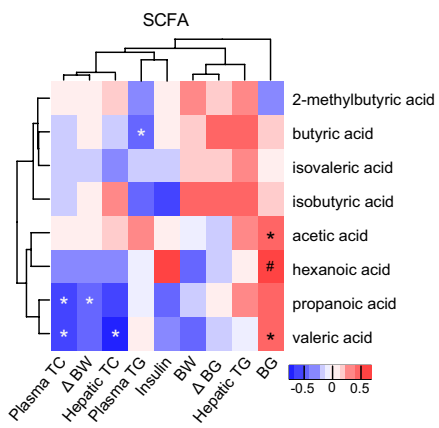

B

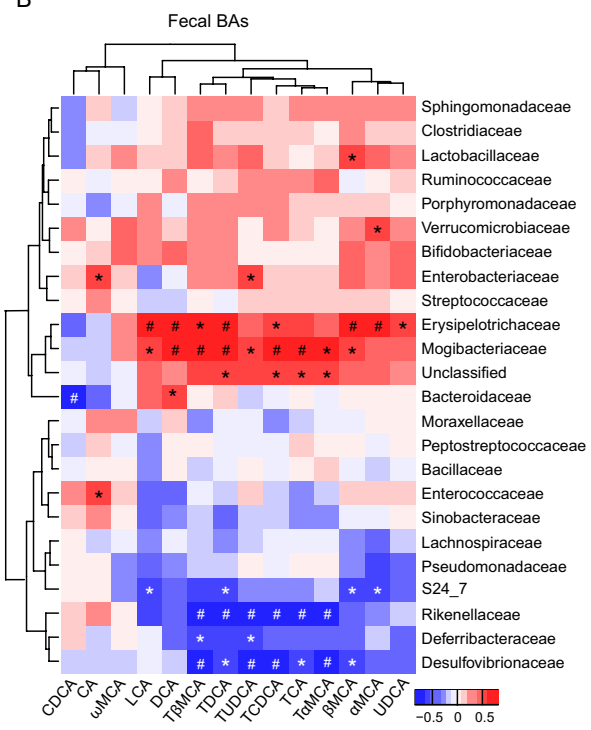

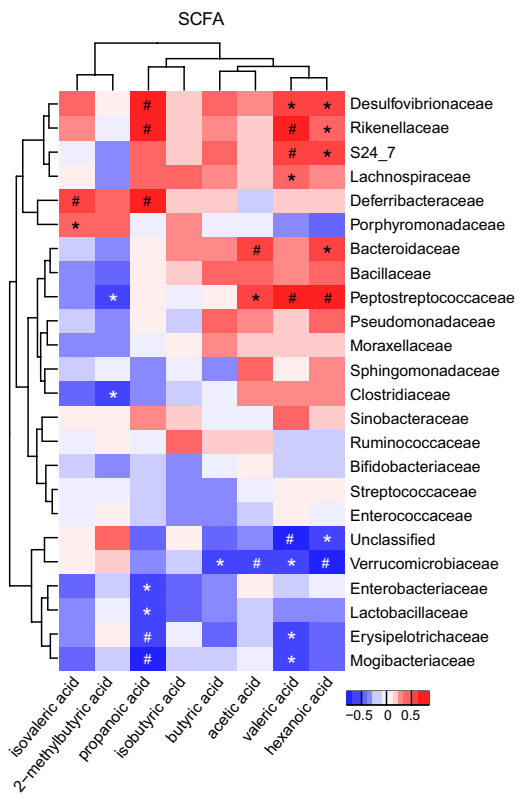

Figure 4

Heatmaps for correlations between gut microbiota, bacterial metabolites and phenotypes. (A) The heatmaps of Spearman's correlation analysis between fecal BAs, SCFAs and mice phenotypes after abx withdrawal. (B) The heatmaps of Spearman's correlation analysis between fecal BAs, SCFAs and RAs of bacterial families after abx withdrawal. The color key represents the values of correlation co-efficiency. CA, cholic acid; CDCA, chenodeoxycholic acid; MCA, murine cholic acid; $\alpha M C A$, alpha MCA; $\beta$ MCA, beta MCA; TCA, taurine conjugated cholic acid; TCDCA, taurine conjugated chenodeoxycholic acid; T $\alpha M C A$, taurine conjugated alpha MCA; T $B M C A$, taurine conjugated beta MCA; $\omega$ MCA, omega MCA; DCA, deoxycholic acid; LCA, lithocholic acid; UDCA, ursodeoxycholic acid; TDCA, taurine conjugated deoxycholic acid; TLCA, taurine conjugated lithocholic acid; TUDCA, taurine conjugated ursodeoxycholic acid; taurine BAs. $n=6-8$ for each group. ${ }^{*} P<0.05,{ }^{*} P<0.01$, Spearman's correlation. abx effects on gut microbiota taxa changes could be responsible for the alterations in microbial BA metabolism and SCFA production, which might exert impacts on metabolic phenotypes of $\mathrm{db} / \mathrm{db}$ mice, such as BW gain, plasma and hepatic TC levels as well as BG variations.

\section{Hepatic BA synthesis and cholesterol transportation were impaired in $\mathrm{db} / \mathrm{db}$ mice with previous $\mathrm{abx}$ treatment}

To gain further insight of how host regulations of BAs and cholesterol associated with the changes from gut microbiota, we next determined the expression levels of key genes involved in BA and cholesterol metabolism of $\mathrm{db} / \mathrm{db}$ mice after abx withdrawal. The mRNA expression levels of hepatic BA synthesis genes, $C y p 7 b 1$ significantly reduced in $\mathrm{p}-\mathrm{Abx}$ mice with a trend of reduced expressions of Cyp7a1, Cyp27a1 as well as Cyp2c70 that controls hepatic murine primary BA synthesis (Takahashi et al. 2016) (Fig. 5A). The expressions of genes related to taurine metabolism and taurine BA conjugation in the liver
(Fig. 5B), including cysteine dioxygenase (Cdo), taurine transporter (Taut), bile acid-CoA synthetase (Bacs), were also reduced. The major BA transporters, liver bile salt export pump (Bsep), organic solute transporter $\alpha$ (Osta) and gut solute carrier family 10, member 2 (Scl10a2 or $A s b t)$, also remarkably reduced their expressions in $\mathrm{p}-\mathrm{Abx}$ (Fig. 5C and D). These results were in line with the increased plasma FGF15 levels (Fig. 5E), which is a major inhibitory signal secreted from gut for regulating hepatic BA synthesis, conjugation, biliary excretion and gut reabsorption of BA (Sayin et al. 2013). Both hepatic FXR and its direct downstream effector, SHP, were repressed in the p-Abx mice (Fig. 5F and G), consistent with the higher hepatic and plasma T $\beta M C A$ percentage, as the potent FXR antagonist (Sayin et al. 2013, Jiang et al. 2015a).

As to the cholesterol metabolism, the gene expressions of the hepatic Niemann-Pick type C1 (Npc1) and sterol transporters such as ATP-binding cassette subfamily A member 1 (Abca1) and ATP-binding cassette subfamily G member $1 / 5(A b c g 1 / 5)$ were also markedly reduced in the p-Abx group $(P<0.05$, Fig. $5 \mathrm{H})$. Whereas the de novo 


\begin{tabular}{l|l|l|r|r|}
$\begin{array}{l}\text { Journal of } \\
\text { Endocrinology }\end{array}$ & L Fu, Y Qiu et al. & $\begin{array}{l}\text { Postpone impacts of antibiotics } \\
\text { on db/db mice }\end{array}$ & $\mathbf{2 3 8 : 2}$ & $\mathbf{1 4 5}$ \\
\hline
\end{tabular}

A
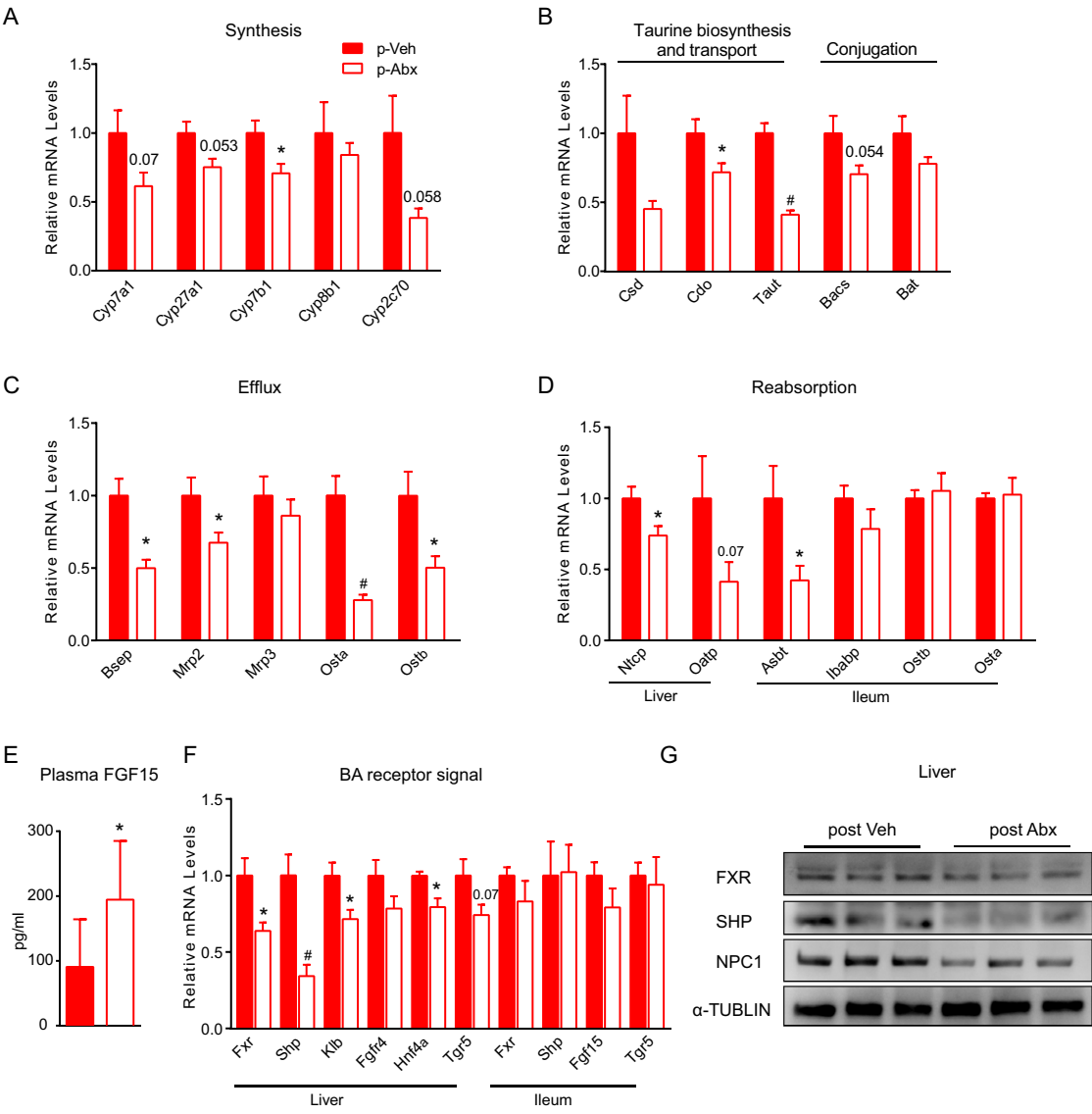

G

B

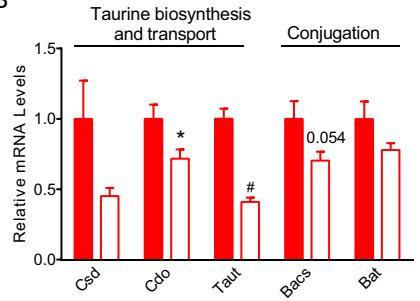
$\alpha-T U B L I N$

\section{I}

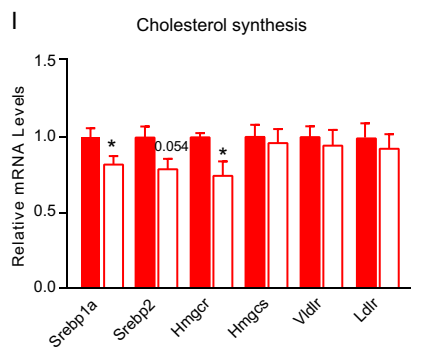

\section{Figure 5}

The expression of genes involved in bile acids and cholesterol metabolism after abx withdrawal. The expression of genes regulating (A) BAs synthesis. (B) Taurine biosynthesis, transport and bile acid conjugation. (C) BAs efflux in liver, and (D) reabsorption in liver and distal ileum. (E) Fasting plasma level of FGF15. (F) BAs receptor and other post $B A$ receptor signals. (G) Western blot for demonstrating hepatic FXR, SHP and NPC1 protein levels. $n=3$ for each group. Gene expression of cholesterol transport in liver and the distal ileum $(\mathrm{H})$, cholesterol biosynthesis (I). $n=6-8$ for each group. Data are showed as mean \pm S.E.M., ${ }^{*} P<0.05,{ }^{\sharp} P<0.01, \mathrm{p}$-Abx vs $\mathrm{p}$-Veh, Kruskal-Wallis test. cholesterol synthesis was inhibited in liver manifesting by downregulated 3-hydroxy-3-methylglutaryl-Coenzyme A reductase (Hmgcr) $(P=0.016$, Fig. 5I) and sterol regulatory element binding transcription factor 1a $(P=0.049$, Fig. 5I). The cholesterol uptake regulated by very low-density lipoprotein receptor (Vldlr) and low-density lipoprotein receptor ( $L d l r)$ were not affected (Fig. 5I), nor were the levels of the intestinal cholesterol transporters Abca1, $A b c g 5 / 8$ and Npc1 like intracellular cholesterol transporter 1 (Npc1l1) (Fig. 5I). We further examined the association between all the detected gene expressions levels and the RAs of fecal gut microbiota and only found that the positive correlations between the RA of Rikenellaceae and the expression levels of liver Abca1 and Npc1 were significant (Spearman correlation, $q<0.05$, Supplementary Fig. 7 and Supplementary Table 2). Thus, in addition to blocking liver BA synthesis, long-lasting effects of abx treatment on gut microbiota could also impede intracellular TC transportation and TC biliary excretion in the liver, via unknown mechanisms, to promote the hepatic TC accumulation.

\section{Discussion}

Exposures to abx in both animal and humans are proved to disrupt metabolism homeostasis in later life and to promote metabolic disorders, including obesity, 
type 1 and 2 diabetes (Ianiro et al. 2016, Livanos et al. 2016, Yassour et al. 2016). This study firstly characterized the long-lasting effects of antibiotics on the metabolic phenotypes, gut microbiota, BAs as well as fecal SCFAs profiles in the $\mathrm{db} / \mathrm{db}$ mice and compared them with the abx transient effects. Our main results showed that abx withdrawal, though can recover many taxa to the control levels changed by the abx treatment, induced a different gut microbiota dysbiosis in $\mathrm{db} / \mathrm{db}$ mice, which might underlie the dysregulation of host cholesterol metabolism via disrupting bacterial BAs and SCFAs metabolism.

The acute responses of gut microbiota to $a b x$ treatment in $\mathrm{db} / \mathrm{db}$ mice were in line with previous reports on other mouse models (Ley et al. 2005, Cho et al. 2012, Hwang et al. 2015). However, weeks after the abx cessation, the gut microbiota in p-Abx mice exhibited decreased Bacteroidetes and increased Firmicutes abundancies that were similar with the feature of gut microbiota in obese subjects (Ley et al. 2006, Ridaura et al. 2013, Hu et al. 2015). The decreased Desulfovibrionaceae (Panasevich et al. 2016) and Rikenellaceae (Daniel et al. 2014) belong to 'leanness' taxa and the increased Erysipelotrichaceae and Mogibacteriaceae are designated as 'obesity' taxa (Kaakoush 2015, Wu et al. 2018). Moreover, abx withdrawal elevated the fecal taurine BAs, plasma FGF15 levels and reduced cecum propionate and valeric acids, which indicated an enhanced gut FXR activity and a lower ability of carbohydrate fermentation of the gut microbiota, as the functional features of obesity-related gut microbiota composition (Ridaura et al. 2013, Jiang et al. 2015b). Hence, the mild gut microbiome dysbiosis induced by delayed abx effects could underlie the rapid catchup BW growth after abx withdrawal in $\mathrm{db} / \mathrm{db}$ mice.
Species under Desulfovibrionaceae, Bilophila wadsworthia, is fermented on taurine deconjugated from PBAs (Devkota et al. 2012) and species under Rikenellaceae, Alistipes putredinis, contains the genes encoding Bsh and BA transporter (Gu et al. 2017). Thus, the declined RA of taxa in both families after abx withdrawal could impair the bacterial $\mathrm{BA}$ deconjugation to increase the fecal taurine BAs. Of note, among the taurine species, T $\beta \mathrm{MCA}$, as the robust FXR antagonist that has been reported to be elevated in both GF- and abx-treated DIO mice to suppress gut FGF15 secretion (Sayin et al. 2013, Jiang et al. 2015a). However, our study found the enhanced fecal T $\beta \mathrm{MCA} \%$ after immediate abx treatment persisted after 24-day abx withdrawal regardless of an elevated plasma FGF15 level. Since the FGF15 is mainly expressed in the ileum and the ileum BA content was not assayed in this study; thus, the fecal T $\beta M C A$ content might not accurately reflect the FXR signaling in the proximal part of gut where the expression of Fgf15 is the highest. It is also possible that changes of other BAs in p-Abx group, such as higher TDCA and lower $\omega \mathrm{MCA}$ (Supplementary Fig. 5D), contribute to the intestinal FXR activity and that the gut FXR signaling regulation might vary between different mouse models. On the other hand, the higher TßMCA levels in liver and plasma were compatible to the attenuated hepatic FXR signaling showed in the results of mRNA expression assessments. The roles of gut BAs or other bacteria metabolites in regulating intestine FXR activity, particularly in diabetes scenario, are worthy to be delineated.

The abx exposure has been reported to impair host cholesterol metabolism and increase cholesterol levels (Carvalho et al. 2012, Nobel et al. 2015). Taxonomically, the positive correlation of Erysipelotrichaceae with hepatic TC is supported by previous studies that have found this taxon decreases with cholesterol-lowering treatment

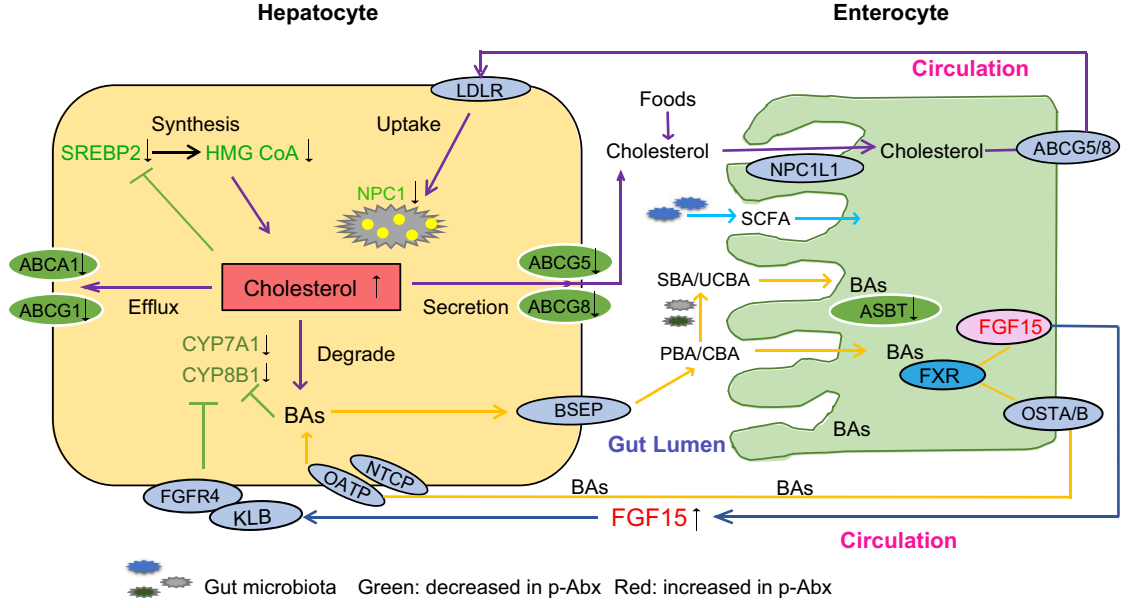

\section{Figure 6}

A proposed molecular mechanism on gut and microbiota interacting of abx postpone impacts on $\mathrm{db} / \mathrm{db}$ mice. After abx withdrawal, BA synthesis was inhibited to recover the enlarged BA pool size by abx treatment, and the ileum Fxr was activated. In addition, cholesterol efflux and clearance in liver were decreased, these all contributed to accumulated cholesterol in liver. 
(Martinez et al. 2009) and in mice with enhanced hepatic conversion of cholesterol to BA exposed in cold ambient temperature (Worthmann et al. 2017). For the metabolites, the decreased cecal propionate level may contribute to the elevated cholesterol levels, for propionate can curb de novo lipid synthesis (Wright et al. 1990, Demigne et al. 1995). More importantly, the elevated plasma FGF15, the decreased expression of genes governing liver BA synthesis, excretion of TC $(A b c g 1 / 5 / 8, A b c a 1)$ and intracellular TC transportation (Npc1), indicated that the hepatic cholesterol metabolism and excretion could be both inhibited to promote the liver cholesterol accumulation in the p-Abx group. The positive correlations of Abca1 and Npc1 expressions with Rikenellaceae supported its associations with TC levels and bacterial metabolites, suggesting that hepatic cholesterol exportation could be targeted by the gut microbiome. Hence, the delayed effect of abx treatment on microbiota could affect microbial SCFAs and BAs metabolism, which might synergistically lower the hepatic cholesterol discharge in $\mathrm{db} / \mathrm{db}$ mice (Fig. 6) and hence elevate hepatic and plasma TC levels. Further study is guaranteed to delineate the regulation mechanism among gut microbiota, microbial metabolites and host cholesterol metabolism.

We observed the BG levels rebounded after abx withdrawal for the recovery of food intake (Supplementary Fig. 3). The Bacteroidaceae, Peptostreptococcaceae and Clostridiaceae were BG levels correlated taxa. Plasma taurine-conjugated cholic acid (TCA), cecal acetic and hexanoic acids were BG correlated bacterial metabolites. The Peptostreptococcaceae, Bacteroidaceae and Clostridiaceae are the main taxa associated with SCFAs (Fig. 4B). Species under Bacteroidaceae have been found correlating positively with BG level and containing the key microbial genes regulating SBA transformation (Gu et al. 2017). Thus, our study might add new input to delineate the bacterial pathways from gut microbiome that might impact the host glucose homeostasis.

In conclusion, our study firstly reported the delayed or long-lasting effects of abx treatment could increase cholesterol levels in the $\mathrm{db} / \mathrm{db}$ mice. The alterations in gut BAs, odd carbon SCFAs as well as in gut microbiota composition we found, suggest a bacterial-host interaction route that mediates the delayed effect of abx to impede the host hepatic cholesterol discharge in T2D. We think our study should raise caution to the usage of abx by studying the microbiota-host interaction in diabetic animal models or further in diabetic patients for preventing the future macrovascular comorbidities, for cholesterol constitutes the main cardiovascular risks in T2D.
Supplementary data

This is linked to the online version of the paper at https://doi.org/10.1530/ JOE-17-0709.

Declaration of interest

The authors declare that there is no conflict of interest that could be perceived as prejudicing the impartiality of the research reported.

\section{Funding}

This work was supported by the National Natural Sciences Foundation of China Grants $(81670761,81570755)$ and the National Basic Research Program of China (2015CB5536003).

\section{Author contribution statement}

$L F, A N$ and $Y G$ conceived and designed the study. $L F, Y Q$ and $L S$ performed the animal experiments. Y G, A N, L F, Y Q, S W and L S analyzed data. X Z performed the LC-MS/MS analyses of plasma bile acid profile. $X$ G performed the GC-MS analyses of cecum SCFA. S W analyzed the gut microbiota data. L F, Y Q, C C, S W and S W prepared figures. L F, $A N$ and $Y G$ wrote the manuscript. $L F, A N, Y G$ and $G N$ edited and revised manuscript. L F, Y Q, L S, C C, Y X, S W, X Z, X G, G N, A N and Y G participated in discussions.

\section{Acknowledgements}

The authors thank Dr Yan Shen (Experimental Animal Center of Ruijin Hospital), Dr Wenyi Li and Jie Yang (Shanghai Institute for Endocrine and Metabolic Diseases, Ruijin Hospital) for animal care. They are grateful to Dr Qidi Wang and Dr Yifei Zhang (Shanghai Institute for Endocrine and Metabolic Diseases, Ruijin Hospital), Dr Hongli Zhang (Department of Endocrine and Metabolic Diseases, The Seventh People's Hospital, Shanghai University of Traditional Chinese Medicine University), Huanzi Zhong and Zhun Shi (BGI Shenzhen), Zhifeng Wang, Xiaoqiang Xu and Xiaokai Wang (Aimigene Institute, Shenzhen) for all the discussions.

\section{References}

Byndloss MX, Olsan EE, Rivera-Chavez F, Tiffany CR, Cevallos SA, Lokken KL, Torres TP, Byndloss AJ, Faber F, Gao Y, et al. 2017 Microbiota-activated PPAR-gamma signaling inhibits dysbiotic Enterobacteriaceae expansion. Science 357 570-575. (https://doi. org/10.1126/science.aam9949)

Carvalho BM, Guadagnini D, Tsukumo DML, Schenka AA, Latuf-Filho P, Vassallo J, Dias JC, Kubota LT, Carvalheira JBC \& Saad MJA 2012 Modulation of gut microbiota by antibiotics improves insulin signalling in high-fat fed mice. Diabetologia 55 2823-2834. (https:// doi.org/10.1007/s00125-012-2648-4)

Cho I, Yamanishi S, Cox L, Methe BA, Zavadil J, Li K, Gao Z, Mahana D, Raju K, Teitler I, et al. 2012 Antibiotics in early life alter the murine colonic microbiome and adiposity. Nature 488 621-626. (https://doi. org/10.1038/nature11400)

Cox LM, Yamanishi S, Sohn J, Alekseyenko AV, Leung JM, Cho I, Kim SG, Li H, Gao Z, Mahana D, et al. 2014 Altering the intestinal microbiota during a critical developmental window has lasting metabolic 
consequences. Cell 158 705-721. (https://doi.org/10.1016/j. cell.2014.05.052)

Daniel H, Gholami AM, Berry D, Desmarchelier C, Hahne H, Loh G, Mondot S, Lepage P, Rothballer M, Walker A, et al. 2014 High-fat diet alters gut microbiota physiology in mice. ISME Journal 8 295-308. (https://doi.org/10.1038/ismej.2013.155)

De Vadder F, Kovatcheva-Datchary P, Goncalves D, Vinera J, Zitoun C, Duchampt A, Backhed F \& Mithieux G 2014 Microbiota-generated metabolites promote metabolic benefits via gut-brain neural circuits. Cell 156 84-96. (https://doi.org/10.1016/j.cell.2013.12.016)

Demigne C, Morand C, Levrat MA, Besson C, Moundras C \& Remesy C 1995 Effect of propionate on fatty acid and cholesterol synthesis and on acetate metabolism in isolated rat hepatocytes. British Journal of Nutrition 74 209-219. (https://doi.org/10.1079/BJN19950124)

Devkota S, Wang Y, Musch MW, Leone V, Fehlner-Peach H, Nadimpalli A, Antonopoulos DA, Jabri B \& Chang EB 2012 Dietary-fat-induced taurocholic acid promotes pathobiont expansion and colitis in Il10-/mice. Nature 487 104-108. (https://doi.org/10.1038/nature11225)

Fujisaka S, Ussar S, Clish C, Devkota S, Dreyfuss JM, Sakaguchi M, Soto M, Konishi M, Softic S, Altindis E, et al. 2016 Antibiotic effects on gut microbiota and metabolism are host dependent. Journal of Clinical Investigation 126 4430-4443. (https://doi.org/10.1172/JCI86674)

Gu Y, Wang X, Li J, Zhang Y, Zhong H, Liu R, Zhang D, Feng Q, Xie X, Hong J, et al. 2017 Analyses of gut microbiota and plasma bile acids enable stratification of patients for antidiabetic treatment. Nature Communications 8 1785. (https://doi.org/10.1038/s41467-017-01682-2)

Hu X, Wang T, Liang S, Li W, Wu X \& Jin F 2015 Antibiotic-induced imbalances in gut microbiota aggravates cholesterol accumulation and liver injuries in rats fed a high-cholesterol diet. Applied Microbiology and Biotechnology 99 9111-9122. (https://doi. org/10.1007/s00253-015-6753-4)

Hwang I, Park YJ, Kim YR, Kim YN, Ka S, Lee HY, Seong JK, Seok YJ \& Kim JB 2015 Alteration of gut microbiota by vancomycin and bacitracin improves insulin resistance via glucagon-like peptide 1 in diet-induced obesity. FASEB Journal 29 2397-2411. (https://doi. org/10.1096/fj.14-265983)

Ianiro G, Tilg H \& Gasbarrini A 2016 Antibiotics as deep modulators of gut microbiota: between good and evil. Gut 65 1906-1915. (https:// doi.org/10.1136/gutjnl-2016-312297)

Jiang C, Xie C, Li F, Zhang L, Nichols RG, Krausz KW, Cai J, Qi Y, Fang ZZ, Takahashi S, et al. 2015 a Intestinal farnesoid X receptor signaling promotes nonalcoholic fatty liver disease. Journal of Clinical Investigation 125 386-402. (https://doi.org/10.1172/JCI76738)

Jiang C, Xie C, Lv Y, Li J, Krausz KW, Shi J, Brocker CN, Desai D, Amin SG, Bisson WH, et al. 2015b Intestine-selective farnesoid X receptor inhibition improves obesity-related metabolic dysfunction. Nature Communications 6 10166. (https://doi.org/10.1038/ ncomms10166)

Kaakoush NO 2015 Insights into the role of erysipelotrichaceae in the human host. Frontiers in Cellular and Infection Microbiology 584. (https://doi.org/10.3389/fcimb.2015.00084)

Ley RE, Backhed F, Turnbaugh P, Lozupone CA, Knight RD \& Gordon JI 2005 Obesity alters gut microbial ecology. PNAS 102 11070-11075. (https://doi.org/10.1073/pnas.0504978102)

Ley RE, Turnbaugh PJ, Klein S \& Gordon JI 2006 Microbial ecology: human gut microbes associated with obesity. Nature 444 1022-1023. (https://doi.org/10.1038/4441022a)

Li J, Jia H, Cai X, Zhong H, Feng Q, Sunagawa S, Arumugam M, Kultima JR, Prifti E, Nielsen T, et al. 2014 An integrated catalog of reference genes in the human gut microbiome. Nature Biotechnology 32 834-841. (https://doi.org/10.1038/nbt.2942)

Livanos AE, Greiner TU, Vangay P, Pathmasiri W, Stewart D, McRitchie S, Li H, Chung J, Sohn J, Kim S, et al. 2016 Antibiotic-mediated gut microbiome perturbation accelerates development of type 1 diabetes in mice. Nature Microbiology 1 16140. (https://doi.org/10.1038/ nmicrobiol.2016.140)
Lynch SV \& Pedersen O 2016 The human intestinal microbiome in health and disease. New England Journal of Medicine 375 2369-2379. (https://doi.org/10.1056/NEJMra1600266)

Magoc T \& Salzberg SL 2011 FLASH: fast length adjustment of short reads to improve genome assemblies. Bioinformatics 27 2957-2963. (https:// doi.org/10.1093/bioinformatics/btr507)

Martinez I, Wallace G, Zhang C, Legge R, Benson AK, Carr TP, Moriyama EN \& Walter J 2009 Diet-induced metabolic improvements in a hamster model of hypercholesterolemia are strongly linked to alterations of the gut microbiota. Applied and Environmental Microbiology 75 4175-4184. (https://doi.org/10.1128/AEM.00380-09)

Mikkelsen KH, Frost M, Bahl MI, Licht TR, Jensen US, Rosenberg J, Pedersen O, Hansen T, Rehfeld JF, Holst JJ, et al. 2015a Effect of antibiotics on gut microbiota, gut hormones and glucose metabolism. PLOS ONE 10 e0142352. (https://doi.org/10.1371/journal. pone.0142352)

Mikkelsen KH, Knop FK, Frost M, Hallas J \& Pottegard A 2015b Use of antibiotics and risk of type 2 diabetes: a population-based case-control study. Journal of Clinical Endocrinology and Metabolism 100 3633-3640. (https://doi.org/10.1210/jc.2015-2696)

Murugesan S, Nirmalkar K, Hoyo-Vadillo C, Garcia-Espitia M, RamirezSanchez D \& Garcia-Mena J 2018 Gut microbiome production of short-chain fatty acids and obesity in children. European Journal of Clinical Microbiology and Infectious Diseases 37 621-625. (https://doi. org/10.1007/s10096-017-3143-0)

Nobel YR, Cox LM, Kirigin FF, Bokulich NA, Yamanishi S, Teitler I, Chung J, Sohn J, Barber CM, Goldfarb DS, et al. 2015 Metabolic and metagenomic outcomes from early-life pulsed antibiotic treatment. Nature Communications 6 7486. (https://doi.org/10.1038/ncomms8486)

Panasevich MR, Morris EM, Chintapalli SV, Wankhade UD, Shankar K, Britton SL, Koch LG, Thyfault JP \& Rector RS 2016 Gut microbiota are linked to increased susceptibility to hepatic steatosis in low-aerobiccapacity rats fed an acute high-fat diet. American Journal of Physiology: Gastrointestinal and Liver Physiology 311 G166-G179. (https://doi. org/10.1152/ajpgi.00065.2016)

Parseus A, Sommer N, Sommer F, Caesar R, Molinaro A, Stahlman M, Greiner TU, Perkins R \& Backhed F 2017 Microbiota-induced obesity requires farnesoid X receptor. Gut 66 429-437. (https://doi. org/10.1136/gutjnl-2015-310283)

Qin J, Li Y, Cai Z, Li S, Zhu J, Zhang F, Liang S, Zhang W, Guan Y, Shen D, et al. 2012 A metagenome-wide association study of gut microbiota in type 2 diabetes. Nature 490 55-60. (https://doi.org/10.1038/ nature11450)

Rabot S, Membrez M, Bruneau A, Gerard P, Harach T, Moser M, Raymond F, Mansourian R \& Chou CJ 2010 Germ-free C57BL/6J mice are resistant to high-fat-diet-induced insulin resistance and have altered cholesterol metabolism. FASEB Journal 24 4948-4959. (https:// doi.org/10.1096/fj.10-164921)

Reijnders D, Goossens GH, Hermes GD, Neis EP, van der Beek CM, Most J, Holst JJ, Lenaerts K, Kootte RS, Nieuwdorp M, et al. 2016 Effects of gut microbiota manipulation by antibiotics on host metabolism in obese humans: a randomized double-blind placebo-controlled trial. Cell Metabolism 24 63-74. (https://doi.org/10.1016/j.cmet.2016.06.016)

Ridaura VK, Faith JJ, Rey FE, Cheng J, Duncan AE, Kau AL, Griffin NW, Lombard V, Henrissat B, Bain JR, et al. 2013 Gut microbiota from twins discordant for obesity modulate metabolism in mice. Science 341 1241214. (https://doi.org/10.1126/science.1241214)

Rios-Covian D, Ruas-Madiedo P, Margolles A, Gueimonde M, de Los Reyes-Gavilan CG \& Salazar N 2016 Intestinal short chain fatty acids and their link with diet and human health. Frontiers in Microbiology 7 185.

Sayin SI, Wahlstrom A, Felin J, Jantti S, Marschall HU, Bamberg K, Angelin B, Hyotylainen T, Oresic M \& Backhed F 2013 Gut microbiota regulates bile acid metabolism by reducing the levels of tauro-betamuricholic acid, a naturally occurring FXR antagonist. Cell Metabolism 17 225-235. (https://doi.org/10.1016/j.cmet.2013.01.003) 
Takahashi S, Fukami T, Masuo Y, Brocker CN, Xie C, Krausz KW, Wolf CR, Henderson CJ \& Gonzalez FJ 2016 Cyp2c70 is responsible for the species difference in bile acid metabolism between mice and humans. Journal of Lipid Research 57 2130-2137. (https://doi.org/10.1194/jlr. M071183)

Turnbaugh PJ, Ley RE, Mahowald MA, Magrini V, Mardis ER \& Gordon JI 2006 An obesity-associated gut microbiome with increased capacity for energy harvest. Nature 444 1027-1031. (https://doi.org/10.1038/ nature05414)

Wahlstrom A, Sayin SI, Marschall HU \& Backhed F 2016 Intestinal crosstalk between bile acids and microbiota and its impact on host metabolism. Cell Metabolism 24 41-50. (https://doi.org/10.1016/j. cmet.2016.05.005)

Worthmann A, John C, Ruhlemann MC, Baguhl M, Heinsen FA, Schaltenberg N, Heine M, Schlein C, Evangelakos I, Mineo C, et al.
2017 Cold-induced conversion of cholesterol to bile acids in mice shapes the gut microbiome and promotes adaptive thermogenesis. Nature Medicine 23 839-849. (https://doi.org/10.1038/nm.4357)

Wright RS, Anderson JW \& Bridges SR 1990 Propionate inhibits hepatocyte lipid synthesis. Proceedings of the Society for Experimental Biology and Medicine 195 26-29. (https://doi.org/10.3181/00379727195-43113)

Wu Y, Chi X, Zhang Q, Chen F \& Deng X 2018 Characterization of the salivary microbiome in people with obesity. PeerJ 6 e4458. (https:// doi.org/10.7717/peerj.4458)

Yassour M, Vatanen T, Siljander H, Hamalainen AM, Harkonen T, Ryhanen SJ, Franzosa EA, Vlamakis H, Huttenhower C, Gevers D, et al. 2016 Natural history of the infant gut microbiome and impact of antibiotic treatment on bacterial strain diversity and stability. Science Translational Medicine 8 343ra381.

Received in final form 17 May 2018

Accepted 31 May 2018 